\title{
Enfermedad de Gaucher y su manejo clínico en el paciente pediátrico
}

\author{
Emilio González Jiménez ${ }^{a}$, María José Aguilar Corderoa, \\ Judit Álvarez Ferre ${ }^{b}$, Pedro Antonio García López ${ }^{c}$
}

\begin{abstract}
a Departamento de Enfermería. Escuela Universitaria de Ciencias de la Salud. Universidad de Granada. Granada.

${ }^{\mathrm{b}}$ Hospital Universitario San Rafael. Granada.

c Departamento de Estadística e Investigación Operativa. Facultad de Ciencias. Universidad de Granada.

Correspondencia: Emilio

González Jiménez,

Departamento de

Enfermería, Escuela

Universitaria de Ciencias de la Salud, Avda. de Madrid s/n, 18071 - Granada, España. Telf.: 6670515 70, correo electrónicol: emigoji@correo.urg.es
\end{abstract}

Recibido el 2 de febrero de 2010.

Aceptado para su publicación el 16 de marzo de 2010.

\begin{abstract}
RESUMEN
La enfermedad de Gaucher constituye una enfermedad lisosomal hereditaria que tiene su origen en un defecto a nivel del gen que codifica el enzima betaglucosidasa ácida, cuya deficiencia condiciona la acumulación de glucocerebrósidos en los lisosomas de macrófagos, causando las manifestaciones clínicas del cuadro. En el niño la forma más frecuente es la tipo 2 o variante neuropática, caracterizada por la presencia de visceromegalias, trastornos hematológicos y alteraciones óseas estructurales. A menudo, presenta mal pronóstico, especialmente cuando sólo se disponía de la esplenectomía y trasplante de medula ósea como únicas terapias. Representa la primera enfermedad en la que se ha utilizado terapia enzimática sustitutiva demostrando su seguridad y eficacia durante la última década, permitiendo mejorar la calidad de vida de los pacientes y disminuyendo su morbimortalidad. El objetivo de este trabajo ha sido mostrar una revisión actualizada acerca de la fisiopatología, diagnóstico y manejo clínico-terapéutico de este complejo proceso.
\end{abstract}

Palabras clave. Enfermedad de Gaucher, Niño.

\section{ABSTRACT}

Gaucher's disease and its clinical management in the pediatric patient

Gaucher disease is an inherited lysosomal disease whose origin lies in a defect at the level of the gene encoding the enzyme acid betaglucosidasa, which deficiency affects the accumulation of glucocerebroside in lysosomes of macrophages, causing the clinical manifestations of the table In children, the most common form is type 2 or neuropathic variant characterized by the presence of visceromegalies, hematological disorders and structural bone changes. Often presents a poor prognosis, especially when they were available splenectomy and bone marrow transplantation as the only therapies. It represents the first disease in which enzyme replacement therapy has been used to demonstrate safety and efficacy over the last decade, enabling improved patients' quality of life and decreasing their morbidity and mortality. The aim of this study was to show an updated review about the pathophysiology, diagnosis and clinical management - treatment of this complex process.

Key words. Gaucher Disease, Child.

\section{INTRODUCCIÓN}

La enfermedad de Gaucher tiene su origen en una deficiencia enzimática, concretamente del enzima glucocerebrosidasa. La carencia de éste enzima provoca una acumulación de glucosilceramida, que procede de la degradación de las membranas celulares en los lisosomas de los macrófagos procedentes de gran cantidad de órganos como huesos, hígado, bazo y médula ósea (células de Gaucher) ${ }^{1}$. Desde un punto de vista genético, su transmisión es autonómica recesiva, es decir, para su transmisión sendos progenitores han de ser portadores de dicha anomalía y en este caso sólo un $25 \%$ de los descendientes podrán padecer la enfermedad. El gen que codifica la enzima se encuentra en el brazo largo del cromosoma 1 (1q21). No obstante, en la actualidad se han descrito más de trescientas mutaciones implicadas en su aparición. Además, dicho trastorno puede ser originado a partir de deleciones, inserciones y por alelos recombinantes ${ }^{2}$. 


\section{EPIDEMIOLOGÍA}

Este trastorno fue descrito por primera vez en 1882 , por el dermatólogo francés Phillipe Charles Gaucher $^{3}$. Años más tarde, en 1965, las investigaciones de Brady ${ }^{4}$ demostraron que la enfermedad de Gaucher estaba producida por el déficit de la enzima lisosómica beta-glucosidasa ácida o cerebrosidasa, responsable de la hidrólisis intracelular de la glucosilceramida y otros esfingolípidos afines. Este trastorno enzimático provocará la acumulación de éstos esfingolípidos a nivel de los lisosomas que integran el sistema retículo endotelial. Por su parte, Groen $(1948)^{5}$ sería el primero en describir la transmisión genética de este complejo trastorno, sugiriendo así que se trataba de una enfermedad con carácter autosómico recesivo y cuya anomalía de base se hallaba localizada en el cromosoma 1 (1q2.1). Desde un punto de vista étnico, se ha podido verificar una mayor prevalencia entre sujetos de determinados grupos étnicos, como es el caso de los Norrbottnian del norte de Suecia entre los que predomina del subtipo III, o los judíos de origen Ashkenazi en donde destaca el tipo I, con una frecuencia de portadores de 1:400 a 1:800 nacidos vivos, a diferencia de la población general cuya frecuencia es de 1:40.000 a 1:60.000 nacidos vivos. No obstante, y a pesar de su relativo carácter étnico, la enfermedad puede afectar por igual a cualquier grupo étnicoracial $^{6}$. En Europa su prevalencia es escasa, siendo descrita una media de cinco casos por cada 10.000 habitantes, lo que se traduciría en menos de 10.000 pacientes en todo el mundo ${ }^{7}$.

\section{FISIOPATOLOGÍA}

En la actualidad, y a pesar de los numerosos avances en su estudio fisiopatológico, no ha sido posible establecer correlaciones fenotipo-genotipo certeras $^{8}$. Las únicas asociaciones genotipo-fenotipo reconocidas son que N370S en un alelo no se relaciona con afectación neurológica y cómo los pacientes con homocigosidad para L444P pueden desarrollar cierto grado de afectación neurológica durante alguna fase de la enfermedad8. Del mismo modo, se desconocen los mecanismos a través de los cuales un determinado genotipo determina un fenotipo concreto. Resulta igualmente inexplicable el hecho de que pacientes portadores de la misma mutación muestren cuadros clínicos diferentes. Circunstancia, ésta última, posiblemente debida a la influencia de otros factores como los ambientales, a la presencia de genes continuos o de modificadores genéticos.

Desde un punto de vista fisiológico y en condiciones de normalidad, la síntesis y destrucción de los glucoesfingolípidos ha de representar un proceso en perfecto equilibrio interno. Los esfingolípidos tienen funciones dentro de las células, por ejemplo, la ceramida y la esfingosina-1-fosfato son segundos mensajeros y adicionalmente inician los mecanismos de apoptosis ${ }^{9}$. La esfingosina también es un segundo mensajero y su generación le permite a la célula sobrevivir. Por su estructura química, aquellos glucoesfingolípidos que poseen más de cuatro unidades de glucosa en su cadena requieren únicamente de enzimas hidrolíticas para su metabolis$\mathrm{mo}^{10}$. En cambio, aquellos otros que tienen menos glucosa en su cadena requieren, además de esta enzima hidrolítica, una segunda molécula del grupo de proteínas activadoras de esfingolípidos, que para la glucocerebrosidasa se llama saposina C. La saposina $C$ es codificada por un gen ubicado en el cromosoma 10 y constituye una proteína activadora de membrana con capacidad para solubilizar los lípidos, y en definitiva resulta responsable de la degradación de las glucosilceramidas ${ }^{11}$.

El proceso de degradación de los glucoesfingolípidos por estas enzimas no es bien conocido por el momento. En la enfermedad de Gaucher el depósito de los glucoesfingolípidos se realiza a nivel de los lisosomas de los macrófagos presentes en el sistema retículoendotelial ${ }^{12}$. Resultado de ello y del aspecto particular que estas células adquieren, determina su apelativo como células de Gaucher. Microscópicamente, dichas células muestran un gran tamaño, portando un núcleo excéntrico y un citoplasma que se asemeja a un papel arrugado ${ }^{13,14}$. En la figura 1 se muestra el aspecto del citoplasma tan característico de las células de Gaucher.

En los pacientes pediátricos el acúmulo de glucocerebrósido no explica el cuadro clínico, en tanto que su cantidad acumulada a nivel de las células del sistema nervioso central no resulta elevada ${ }^{15}$. Se cree más bien que se trate pues de un metabolito tóxico denominado glucoesfingosina, cuya concentración se encuentra elevada en el hígado y bazo de los pacientes portadores de este trastorno. Dicha acumulación tiene su inicio tempranamente durante la gestación. En este caso, niveles elevados de esta sustancia en el cerebro se encuentra únicamente en pacientes de corta edad portadores de la variedad tipo $2^{16}$.

Posteriormente, derivado de la acumulación de grandes cantidades de glucoesfingolípidos en el interior de las células, pueden producirse interferencias a nivel del transporte intracelular y otras actividades de la célula. En el caso de la enfermedad de Gaucher, la psicosina es la sustancia tóxica derivada del glucocerebrósido acumulado ${ }^{17}$.

Las manifestaciones clínicas de la enfermedad resultan muy variadas dada la heterogeneidad del defecto genético subyacente, constituyendo un reflejo del acumulo de material procedente de las membranas celulares de las células sanguíneas envejecidas no degradado. En la forma neuropática la sintomatología es resultado de la degradación incompleta de los gangliósidos a nivel del SNC ${ }^{18}$. 
En general, las glucoesfingolipidosis se pueden clasificar en tres grandes grupos dependiendo de la población a la que afecten. Así podemos hablar de una glucoesfingolipidosis infantil, juvenil y del adulto ${ }^{19}$. Por su pronóstico, la variante infantil ostenta el peor pronóstico, ya que en los adultos la cantidad residual de enzima existente posibilita que los síntomas sean menos intensos y que la expectativa de vida sea mayor ${ }^{19}$.

La enfermedad de Gaucher (EG), tal y como se ha mencionado, se caracteriza por una gran heterogeneidad en cuanto a sus manifestaciones clínicas, pudiendo ser asintomática o por el contrario tan grave que produzca la muerte ${ }^{20}$. Con todo ello, se sabe cómo la intensidad de las manifestaciones clínicas no se correlaciona de manera estrecha con el nivel de actividad enzimática ni tampoco con la mutación concreta presente en el enfermo. La expresión clínica del cuadro estará determinada por la interacción de múltiples factores como la cantidad residual de enzima, el genotipo ${ }^{21}$, factores ambientales y probablemente por la presencia de otras mutaciones no bien conocidas ${ }^{22}$. De este modo y a pesar del considerable grado de heterogeneidad fenotípica, en la actualidad se definen tres variantes clínicas de presentación de la enfermedad ${ }^{23}$.

Variante tipo 1: variante no neuropática, crónica, del adulto.

Variante tipo 2: variante neuropática aguda.

Variante tipo 3: con afectación neurológica y visceral.

Si bien, centrándonos en la variante tipo 2, también denominada variante neuropática aguda, ésta representa la forma menos frecuente (menos del 5\% de los casos). Se manifiesta muy tempranamente, entre el nacimiento y los seis primeros meses de vida, acompañándose de falta de medro, hepatoesplenomegalia y afectación grave y progresiva de SNC (básicamente de las funciones del tallo cerebral, como hipertonía progresiva, estrabismo y retroflexión de la cabeza). La fisiopatología no es la misma que para la forma visceral, ya que en los especímenes de cerebro no se ha encontrado abundante acúmulo de glucorebrósido para explicar el daño, por lo que se considera que el daño se da por muerte neuronal.

La glucocerebrosidasa degrada igualmente otro sustrato, la glucosilesfingosina en glucosa y esfingosina $^{19}$. La glucosilesfingosina es una sustancia neurotóxica que puede producir alteración de la actividad celular normal con interrupción de la transmisión de señales por inhibición de la proteína cinasa $\mathrm{C}$, de la diferenciación celular y posiblemente de los mecanismos de muerte celular programada. La acumulación de glucoesfingolípidos y disminución de moléculas de señalización producen alteración de la transmisión de señales, respuestas celulares defectuosas y lleva a diferentes síntomas y diversidad en la patogénesis.

En la clínica, las manifestaciones neurológicas cursan con retraso del desarrollo, pérdida de habilidades adquiridas, convulsiones y movimientos oculares sacádicos (desplazamientos oculares de un punto a otro a alta velocidad), cuya función es traer la imagen desde la periferia hasta el centro de la retina, donde la agudeza visual resulta mayor ${ }^{22}$. Además, estos pacientes desarrollan alteraciones bulbares con estridor y dificultad para la deglución, signos de afectación extrapiramidal con aumento del tono muscular, opistótonos, cabeza con retroflexión y espasticidad. Resultado de ello, en su mayoría suelen fallecer antes del segundo año de vida como consecuencia de complicaciones pulmonares secundarias al daño neurológico ${ }^{22}$.

También puede haber afectación cutánea en forma de ictiosis, que puede ser congénita, con muerte temprana en las primeras semanas de vida, o de aparición tardía y muy grave (niño en colodión), por lo que la EG es uno de los diagnósticos que se debe considerar en los niños con ictiosis congénita. Se ha descrito una forma perinatal letal con esplenomegalia masiva y progresiva, hipotonía, falla renal e hiperferritinemia sin afectación cutánea. También, una forma de iniciación más tardía en la que se desarrolla estrabismo en el primer año de vida, pero el niño continúa bien hasta el segundo o tercer año de edad, cuando se manifiesta floridamente la enfermedad.

\section{COMPLICACIONES EN EL NIÑO}

Las complicaciones de la enfermedad de Gaucher son múltiples. Sin embargo, las más frecuentes son las secuelas óseas ${ }^{24}$. Estas pueden ser desde un faIlo asintomático a nivel del proceso de remodelado óseo (deformidad en forma de matraz de Erlenmeyer), hasta infartos medulares, fracturas vertebrales, osteonecrosis de las cabezas femoral y humeral y colapso vertebral. La deformidad en forma de matraz de Erlenmeyer está presente en el $80 \%$ de los pacientes, siendo la región distal del fémur y la proximal de la tibia las zonas de presentación más comunes. En la figura 2 se muestra la deformidad en forma de matraz de Erlenmeyer.

Si bien, otras menos frecuentes son igualmente importantes. Así, desde un punto de vista hepático, pueden desarrollar várices esofágicas con el consiguiente desarrollo de hipertensión portal, secundaria a cirrosis hepática. La colelitiasis no es un hallazgo frecuente en pacientes con enfermedad de Gaucher, sin embargo parece ser poco reconocida. Puede deberse a anemia crónica, antecedente de esplenectomía, excreción biliar de glucosilceramida y antecedente de afectación hepática, así como cirrosis. Del mismo modo, estos pacientes pueden 
desarrollar cuadros infecciosos piógenos graves derivados de la leucopenia, además de la disfunción esplénica. Por su parte, el absceso esplénico es una complicación rara en niños. Además, algunos pacientes pueden desarrollar complicaciones como el síndrome nefrótico o neoplasias hematolinfoides (linfomas) y mieloma múltiple ${ }^{25,26}$.

\section{DIAGNÓSTICO}

Hasta 1980 el diagnóstico de esta enfermedad era realizado únicamente a partir de datos morfológicos, esto es, identificar histológicamente las células de Gaucher. Estas células, que no son patognomónicas, se ven con relativa facilidad en fresco en aspirados de bazo y de médula ósea.

Por su parte, la determinación de la actividad deficiente de la enzima glucocerebrosidasa $ß$ resulta fundamental para alcanzar su diagnóstico. Esta actividad deficiente se puede dosificar en leucocitos y cultivos de fibroblastos. Las pruebas pueden hacerse en sangre completa o en gotas de sangre seca, una técnica que permite el estudio con poca cantidad de muestra y en centros de referencia que pueden estar muy lejos del paciente. Los sujetos afectados se distinguen muy bien de los portadores y de los sujetos normales por la disminución de actividad enzimática, constituyendo un método seguro para el diagnóstico de la enfermedad. En el caso de los portadores y los sujetos normales la situación es diferente, pues se produce un solapamiento entre actividad de portadores y no portadores que no permite establecer la distinción por este procedimiento. Tampoco permite distinguir entre los diferentes tipos de la enfermedad, ya que todos los pacientes tienen actividad por debajo de $10 \%$. Ahora bien, existen determinados marcadores bioquímicos con capacidad para mantener un seguimiento de la actividad de la enfermedad, como la enzima quitotriosidasa que es útil siempre que el paciente no presente una deficiencia congénita $(6 \%$ de la población general la padece $)^{27}$. Esta enzima se produce en macrófagos activados y su actividad está incrementada en el suero de pacientes con enfermedad de Gaucher.

Otra de las pruebas complementarias útiles en el diagnostico de EG es la determinación de la Fosfatasa ácida resistente al tartrato, la cual se incrementa en las células de Gaucher. También, la enzima convertidora de angiotensina (ACE), que aumenta en los pacientes con enfermedad de Gaucher. Por último, la fosfatasa alcalina que puede elevarse en aquellos pacientes con afección ósea ${ }^{26}$.

Otros procedimientos diagnósticos igualmente útiles son las imágenes radiológicas de los huesos y vísceras afectados ${ }^{28}$. Así, las radiografías simples de huesos ayudan a demostrar lesiones ya establecidas, como la deformidad en Erlenmeyer, lesio- nes líticas, infartos, necrosis avascular, fracturas y osteopenia ${ }^{29},{ }^{30}$. La resonancia magnética, por su parte, resulta una técnica muy útil para la detección de afectación ósea temprana tal como la infiltración medular, tanto en huesos largos como en vértebras y para detectar signos tempranos de infartos óseos precedentes de necrosis avasculares ${ }^{31}$. La densitometría ósea por DEXA es necesaria, ya que muchos niños con esta enfermedad tienen alteraciones de la densidad mineral ósea al diagnóstico, y ésta puede influir en la velocidad de crecimiento de los niños originando un retardo en el mismo ${ }^{31,32}$. En cuanto a la evaluación visceral, la afectación es básicamente de hígado y bazo, y puede hacerse por ultrasonido, tomografía axial computarizada o resonancia magnética ${ }^{33}$. En el hígado se pueden encontrar signos de crecimiento visceral, nódulos e hipertensión portal. En el bazo, además del crecimiento visceral, se pueden encontrar lesiones focales de infartos o de abscesos.

\section{TRATAMIENTO EN EL PACIENTE PEDIÁTRICO}

Dependiendo de los síntomas y del estado de desarrollo, la terapia de la enfermedad de Gaucher resultará variable. Así, incluye medidas como el reposo en cama y la utilización de analgésicos, aunque nunca "aspirina" (inhibe la coagulación de la sangre y no es recomendable para la gente con la enfermedad de Gaucher). Otras medidas utilizadas son el uso de antiinflamatorios para el dolor agudocrónico y la terapia con oxígeno hiperbárico para el tratamiento de las crisis agudas de los huesos. La terapia de oxígeno puede ser necesaria para aquellos pacientes con una cantidad reducida de sangre en los pulmones. Los bajos niveles de sangre originados por una hiperactividad esplénica conteniendo células de Gaucher a menudo son tratados con transfusiones de sangre y/o plaquetas, al mismo tiempo que con terapia de hierro para corregir la anemia. En casos de disminución severa y persistente de los niveles de la sangre, cabe contemplar la posible extirpación total o parcial del bazo ${ }^{34}$.

Sin embargo, ninguno de estos métodos es totalmente satisfactorio. En su caso, la terapia con hierro aumenta el riesgo a la hemocromatosis, mientras que la extirpación del bazo aumenta la susceptibilidad a las enfermedades bacterianas y puede conducir a un aumento de los síntomas a nivel del hígado y del esqueleto óseo. Por todo ello, la extirpación total del bazo (no la parcial) suele contemplarse como última opción, retrasándola lo máximo posible.

Si bien, entre las posibles opciones terapéuticas, la que mejores perspectivas ha mostrado, por el momento, es la terapia de reemplazo enzimático (TRE) ${ }^{35,36}$. Desde su aprobación en 1991 por la FDA, su uso en la práctica clínica habitual ha modificado la historia natural de esta enfermedad. En 
su inicio se obtenía a partir de placentas humanas y posteriormente a partir de tecnología ADN recombinante, siendo el nombre de su principio activo imiglucerasa $^{37,38}$

Actualmente son más de 3.500 los pacientes que han recibido tratamiento con TRE, demostrando una alta eficacia en el control y evolución de la enfermedad ${ }^{35}$. De este modo, la disminución de las visceromegalias se observa en 1 a 3 años, las alteraciones celulares plasmáticas se resuelven en 1 a 5 años y las lesiones óseas reversibles como osteopenia y osteoporosis mejoran en una media de dos años. Algunas lesiones focales en los adultos también mejoran. La terapia de reemplazo enzimático ha sido especialmente efectiva entre pacientes pediátricos con afectación ósea, ya que no sólo puede detener la progresión de la enfermedad sino también inducir a la regresión de algunas de las lesiones preexistentes ${ }^{36}$. Actualmente, se ha establecido una dosificación individual según la extensión de la enfermedad. En este sentido, una vez conocido el grado de afectación orgánica, se deben establecer los objetivos terapéuticos para en base a ello establecer la dosis óptima. En niños, se recomienda siempre iniciar el tratamiento con dosis no superiores a 40 a $60 \mathrm{U} / \mathrm{kg}$. Su continuo estado de crecimiento implica modular la dosis, así como también para evitar el agravamiento de las lesiones óseas, ya que con la aplicación del fármaco a dosis altas se corre el riesgo de empeorar las lesiones óseas ya existentes ${ }^{37}$. Habitualmente se administra una dosis cada quince días, con evaluaciones periódicas (según el consenso elegido para su seguimiento) para realizar los ajustes de dosis necesarios. El tratamiento desde su inicio tendrá carácter indefinido dado que, según la evidencia científica, suspensiones repentinas en el tratamiento pueden resultar perjudiciales con la consiguiente reaparición de los signos de la enfermedad.

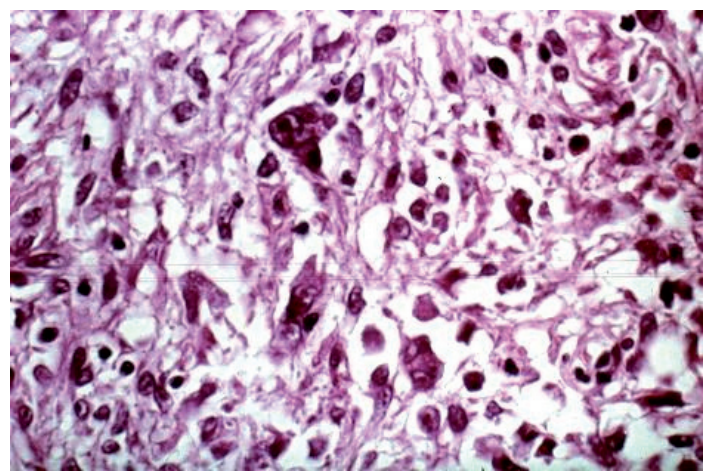

Figura 1. Aspecto del citoplasma de las células de Gaucher.
Otra alternativa terapéutica para estos pacientes son los denominados inhibidores de sustrato. La acción de estos fármacos se fundamenta en la inhibición de la formación del esfingolípido, sin embargo, éstos no son capaces de atravesar la barrera hematoencefálica. De estas moléculas, la OGT-918 ha demostrado mejoría en muchos de los pacientes con enfermedad de Gaucher. Sin embargo, hasta la fecha, dados sus efectos colaterales, su terapia sólo está indicada para aquellos pacientes que tengan contraindicación o problemas para recibir TRE, y sólo en Europa e Israel.

Otros fármacos utilizados en su tratamiento son los bifosfonatos, especialmente para aquellos pacientes con enfermedad ósea ${ }^{38}$, y los vasodilatadores para pacientes con afectación pulmonar. Como alternativa terapéutica a la farmacológica en pacientes con enfermedad neurológica, puede plantearse el trasplante de médula ósea. No obstante, son pocos los pacientes que han recibido estos tratamientos, lo mismo que la terapia génica ${ }^{39}$, aún en etapa de experimentación.

\section{DISCUSIÓN/CONCLUSIÓN}

A pesar de constituir la enfermedad de depósito lisosomal más frecuente en el niño, en la actualidad aún existe cierto grado de controversia respecto de su abordaje clínico. En lo referente a su tratamiento, hemos de destacar cómo la terapia mediante sustitución enzimática ha supuesto un importante avance en la corrección de la alteración metabólica que la origina. Del mismo modo, otros procedimientos como la administración de plasma no fraccionado o de leucocitos, inyecciones de enzima purificada, placenta o bazo, así como la implantación de una fuente de producción enzimática, esto es, el trasplante de fibroblastos de células amnióticas epiteliales y trasplante de riñón, hígado y bazo,

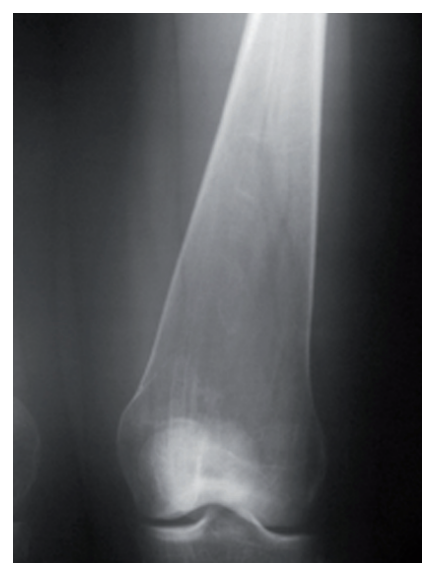

Figura 2. Deformidad en región distal del fémur en forma de matraz de Erlenmeyer. 
han supuesto avances importantes en su manejo y corrección ${ }^{40}$. A pesar de ello, la evidencia científica muestra cómo algunos de estos tratamientos no han resultado ser eficaces para determinadas manifestaciones clínicas, especialmente en aquellas formas clínicas que afectan al sistema nervioso central.

La terapia de reemplazo enzimático con la utilización de glucocerebrosidasa ha supuesto otro importante avance en su tratamiento, mostrando resultados alentadores en el paciente pediátrico. El trasplante de médula ósea (TMO), por su parte, ha sido considerado beneficioso desde su implantación como técnica, pues cabe considerarlo como el preludio para la terapia génica.

Ahora bien, en el caso de ausencia de respuesta a los distintos tratamientos, el abordaje de esta enfermedad resultaría básicamente sintomático, circunstancia de especial interés cuando se trata de pacientes pediátricos. Dada la gravedad del cuadro y la controversia sobre la efectividad de las diferentes opciones terapéuticas, resulta de vital importancia la prevención de nuevos casos, especialmente entre los miembros de familias afectadas en alguno de sus miembros. Dicha prevención tendrá lugar a través del consejo genético. No obstante, para ello será esencial un previo diagnóstico bioquímico para a partir de ese momento contar con la información necesaria sobre el tipo de herencia, riesgo de recurrencia, desarrollo clínico más probable y disponibilidad de metodología para detectar heterocigotos portadores y para el diagnóstico prenatal, para lo cual se emplean células de líquido amniótico cultivadas o biopsia de vellosidades de corion.

Sólo mediante el profundo conocimiento de su fisiopatología, así como de las posibles causas que inducen a su aparición en los pacientes, estaremos en situación de mejorar su abordaje clínico y terapéutico. Nuestro deseo con este trabajo es contribuir con una revisión actualizada del tema y promover la investigación de este cuadro, dado que su abordaje terapéutico parece no quedar claro de modo tácito aún en el presente

\section{BIBLIOGRAFÍA}

1. Charrow J, Esplin JA, Gribble TJ et al. Gaucher disease: recommendations on diagnosis, evaluation, and monitoring. Arch Intern Med. 1998; 158:1754-60.

2. Goker-Alpan O, Schiffmann R, Park JK et al. Phenotypic continuum in neuronopathic Gaucher disease: an intermediate phenotype between type 2 and type 3 . J Pediatr. 2003; 143:2753-63.

3. Gaucher PCE. De l'épithélioma primitif de la rate, hypertrophie idiopathique de la rate sans leucémie, Paris, France: Faculté de Médecine de Paris; 1882. MD Thesis.

4. Brady RO, Kanfer J, Shapiro D. Metabolism of gluco- cerebrosides II. Evidence of an enzymatic deficiency in Gaucher's disease. Biochem Biophys Res Commun. $1965 ; 18: 221-5$.

5. Groen JF. The hereditary mechanism of Gaucher's disease. Blood. 1948; 3:1238-49.

6. Kolodny EH, Charria-Ortiz G. Storage diseases of the reticuloendothelial system. En: Nathan DG, Orkin SH, Ginsburg D, Look AT. Nathan and Oski's Hematology of infancy and childhood. $6^{\mathrm{a}}$ ed. EUA: WB Saunders; 2003. p.1399-54.

7. Hollak CE, Maas M, Aerts M. Clinically relevant therapeutic endpoints in type I Gaucher disease. J Inherit Metab Dis. 2001; 24(Suppl 2):97-105.

8. Ozkara HA. Recent advances in the byochemistry and genetics of sphingolipidoses. Brain Dev. 2004; 26(8):497-505.

9. Niederau C, Haussinger D. Gaucher's disease: a review for the internist and hepatologist. Hepatogastroenterology. $2000 ; 47(34): 984-97$

10. Pastores GM, Weinreb NJ, Aerts $\mathrm{H}$ et al. Therapeutic goals in the treatment of Gaucher Disease. Semin Hematol. 2004; 41(Suppl 5):4-14.

11. Sidransky E. Gaucher disease: complexity in a "simple" disorder. Mol Genet Metab. 2004; 83(1-2): 6-15.

12. Vellodi A, Bembi B, de Villemeur TB et al. Management of neuronopathic Gaucher disease: a European consensus. J Inherit Metab Dis. 2001; 24(Suppl 2):319-27.

13. Beutler E. Gaucher disease: New molecular approaches to diagnosis and treatment. Science. 1992; 256:794-9.

14. Perez-Calvo JI, Pastores GM, Isol AL, Giraldo P, BuenoGomez JM. Enfoque terapéutico de la enfermedad de Gaucher tipo 1. Sangre. 1994; 39:39-44.

15. Adarraga MD, Fernández de la Puebla R, Jiménez JA, Pocoví M, Zambrana JL, Pérez F. Enfermedad de Gaucher. Aportación de 4 casos. Rev Clin Esp. 2002; 202:635-7.

16. Meikle PJ, Hopwood JJ, Clague AE et al. Prevalence of lysosomal storage disorders. JAMA. 1999; 28:249-54.

17. Charrow J, Andersson HC, Kaplan P, Kolodny EH, Mistry P, Pastores G, et al. The Gaucher registry: demographics and disease characteristics of 1698 patients with Gaucher disease. Arch Intern Med. 2000; 160:2835-43.

18. Pérez-Calvo JI, Giraldo P, Rubio-Félix D, Giralt M, Pocoví M. Estudio de calidad del diagnóstico y seguimiento de los trastornos esqueléticos asociados a la enfermedad de Gaucher tipo 1 en España. An Med Interna. 2003; 20:607-11.

19. Grabowski GA. Gaucher disease. Enzymology, genetics, and treatment. Adv Hum Genet. 1993; 21:377-441.

20. Mistry PK. Genotype/phenotipe correlations in Gaucher's disease. Lancet. 1995; 346:982-3.

21. Hruska KS, LaMarca ME, Scott R, Sidransky E. Gaucher Disease: Mutation and Polymorphism Spectrum in the Glucocerebrosidase Gene (GBA). Human Mutation. 2008; 29(5):567-83.

22. Giraldo P, Pérez Calvo JI, Giralt M. Características clínicas de la enfermedad de Gaucher en España. Resultados preliminares de una encuesta nacional. Med Clín (Barc). 1997; 109:619-22.

23. Espin JA, McPherson EJ. Treatment of bone complications in patients with Gaucher disease. Gaucher Clin Perspect. 1994; 2:7-11.

24. Hughes D, Capellini MD, Berger M, et al. Recommendation for the management of the haematological and onco-haematological aspects of Gaucher disease. $\mathrm{Br} \mathrm{J}$ Haematol. 2007; 138: 673-5.

25. Mistry PK, Sadan S, et al. Consequences of diagnostics delays in type 1 Gaucher disease. The need for greater 
awareness among Hematologists-Oncologists and an opportunity for early diagnosis and intervention. Am J Hematology. 2007; 82:697-701.

26. Cox TM. Biomarkers in lysosomal storage diseases: a review. Acta Pædiatrica. 2005; 94(Suppl 447):39-42.

27. Rademakers RP. Radiologic evaluation of Gaucher bone disease. Semin Hematol. 1995; 32 (Suppl. 1):14-19.

28. Wenstrup RJ, Roca-Espiau M, Weinreb NJ, Bembi B. Skeletal aspects of Gaucher disease: a review. Br J Radiol. 2002; 75(Suppl 1):2-12.

29. Maas M, Poll LW, Terk MR. Imaging and quantifying skeltal involvement in Gaucher disease. Br J Radiol. 2002; 75(Supl.1):13-24.

30. Poll LW, Koch JA, vom Dahl S, Willers R, Scherer A, Boerner D, et al. Magnetic resonance imaging of bone marrow changes in Gaucher disease during enzyme replacement therapy: first German long-term results. Skeletal Radiol. 2001; 30:496-503.

31. Hainaux B. Gaucher disease: plain radiography, US, CT and MR diagnosis of lung, bone and liver lesions. Pediatr Radiol. 1992; 22:78.

32. Maas M, Hollak CE, Akkerman EM, Aerts JM, Stoker J, Den Heeten GJ. Quantification of skeletal involvement in adults with type 1 Gaucher's disease: fat fraction measured by Dixon quantitative chemical shift imaging as a valid parameter. Am J Roentgenol. 2002; 179:961-5.

33. Minkes RK, Lagzdins M, Langer JC. Laparoscopic versus open splenectomy in children. J Ped Surg. 2000; 35:699-701.
34. Pérez Calvo Jl. Tratamiento enzimático sustitutivo. En: Giraldo MP, Giralt M, Pérez Calvo JI, Pocoví M, Eds. Enfermedad de Gaucher $2^{\mathrm{a}}$ edición: aspectos generales, clínica, diagnóstico, tratamiento, aspectos psicológicos y calidad de vida. La enfermedad de Gaucher en Iberoamérica. Zaragoza: Ibargüen; 2003. p. 205-22.

35. Rosenthal DI, Doppelt SH, Mankin HJ, Dambrosia JM, Xavier RJ, McKusick KA, et al. Enzyme replacement therapy for Gaucher's disease: skeletal responses to macrophage-targeted glucocerebrosidase. Pediatrics. 1995; 96:629-37.

36. Andersson HC, Charrow J, Kaplan P, Mistry P, Pastores GM, Prakesh-Cheng A, et al. Individualization of longterm enzyme replacement therapy for Gaucher disease. Genet Med. 2005:7(2):105-10.

37. Weinreb NJ, Charrow J, Andersson HC, Kaplan P, Kolodny EH, Mistry P, et al. Effectiveness of enzime replacement therapy in 1028 patients with type 1 Gaucher disease after 2 to 5 years of treatment: a report from the Gaucher Registry. Am J Med. 2002; 113:112-9.

38. Srivastava T, Alon US. The role of bisphosphonates in diseases of childhood. Eur J Pediatr. 2003; 162:735-51.

39. Alfonso P, Aznarez S, Giralt M, Pocoví M, Giraldo P. Mutation analysis and genotype/phenotype relationships of Gaucher disease patients in Spain. J Hum Genet. 2007; 52:391-6.

40. Brooks DA. Immune response to enzyme replacement therapy in lysosomal storage disorder patients and animal models. Molec Genet Metab. 1999; 68:268-75. 